Editorial

\title{
Acknowledgement to Reviewers of Future Internet in 2016
}

\section{Future Internet Editorial Office}

Published: 12 January 2017

MDPI AG, St. Alban-Anlage 66, 4052 Basel, Switzerland; futureinternet@mdpi.com

The editors of Future Internet would like to express their sincere gratitude to the following reviewers for assessing manuscripts in 2016.

We greatly appreciate the contribution of expert reviewers, which is crucial to the journal's editorial process. We aim to recognize reviewer contributions through several mechanisms, of which the annual publication of reviewer names is one. Reviewers receive a voucher entitling them to a discount on their next MDPI publication and can download a certificate of recognition directly from our submission system. Of course, in these initiatives we are careful not to compromise reviewer confidentiality. Many reviewers see their work as a voluntary and often unseen part of their role as researchers. We are grateful to the time reviewers donate to our journals and the contribution they make.

If you are interested in becoming a reviewer for Future Internet, see the link at the bottom of the webpage http://www.mdpi.com/reviewers.

The following reviewed for Future Internet in 2016:

$\begin{array}{lll}\text { Abanda, Henry } & \text { Cai, Guoray } & \text { Doulamis, Nikolaos D. } \\ \text { Adelantado, Ferran } & \text { Calanca, Pierluigi } & \text { Esposito, Christian } \\ \text { Ahmad, Awais } & \text { Camarinha-Matos, Luis M. } & \text { Fahrnberger, Günter } \\ \text { Al Mtawa, Yaser } & \text { Casado, Rubén } & \text { Feld, Sebastian } \\ \text { Alasaad, Amr } & \text { Cattaneo, Giuseppe } & \text { Fesenmaier, Daniel R. } \\ \text { Almenárez, Florina } & \text { Cela, Manuel } & \text { Ficco, Massimo } \\ \text { Al-Sherbaz, Ali } & \text { Chang, Hangbae } & \text { Gangeh, Mehrdad J. } \\ \text { Álvarez-García, José } & \text { Chen, Lieu-Hen } & \text { Gao, Chunming } \\ \text { Andersen, Anders } & \text { Cherdantseva, Yulia } & \text { Garaizar, Pablo } \\ \text { Andersson, Karl } & \text { Choo, Raymond } & \text { García-Peñalvo, Francisco J. } \\ \text { Antonini, Andrea } & \text { Chung, Chun-Jen } & \text { Gebremariam, Anteneh A. } \\ \text { Anttiroiko, Ari-Veikko } & \text { Comyn-Wattiau, Isabelle } & \text { Gibon, Annick } \\ \text { Arazy, Ofer } & \text { Conclio, Grazia } & \text { Glynn, Peter J. } \\ \text { Baharon, Mohd Rizuan } & \text { Conde-González, Miguel Ángel } & \text { Gunnell, David } \\ \text { Barceló, Marc } & \text { Cord, Anna } & \text { Guzman, Javier Garcia } \\ \text { Barkaoui, Kamel } & \text { Costa-Requena, Jose } & \text { Haller, Armin } \\ \text { Benninger, Daniel } & \text { Cozza, Vittoria } & \text { He, Ying } \\ \text { Bick, Markus } & \text { Delmastro, Franca } & \text { Herrero Gutierrez, Francisco J. } \\ \text { Bonaiuti, Giovanni } & \text { Desprats, Thierry } & \text { Hill, Benjamin Mako } \\ \text { Brewer, Peter W. } & \text { Dias, Francisco } & \text { Hodgson, Tim } \\ \text { Brownlee, Nevil } & \text { Diraco, Giovanni } & \text { Hsieh, Jui-Chien } \\ & & \end{array}$


Inácio, Pedro R. M.

Iñiguez, Gerardo

Iracleous, Dimitrios P.

Jansen, Slinger

Jaramillo, Patricia A.

Jiang, Yifei

Jung, Jason J.

Kameoka, Takaharu

Kamsu-Foguem, Bernard

Kankanhalli, Mohan S.

Katsikas, Sokratis

Kidholm, Kristian

Kieseberg, Peter

Kikis-Papadakis, Kathy

Kim, Kiil

Knöll, Martin

Koien, Geir M.

Krotofil, Marina

Lagrange, Xavier

Laurent, Maryline

Le, Hieu Hanh

Lee, Chao-Hsien

Lee, Gyu Myoung

Lee, Malrey

Lee, Tae-Gyu

Lee, Won Cheol

Leligou, Helen C.

Lloret-Mauri, Jaime

Lombardi, Flavio

Maglaras, Leandros

Manca, Stefania

Marrone, Stefano

Matos, Ana Raquel

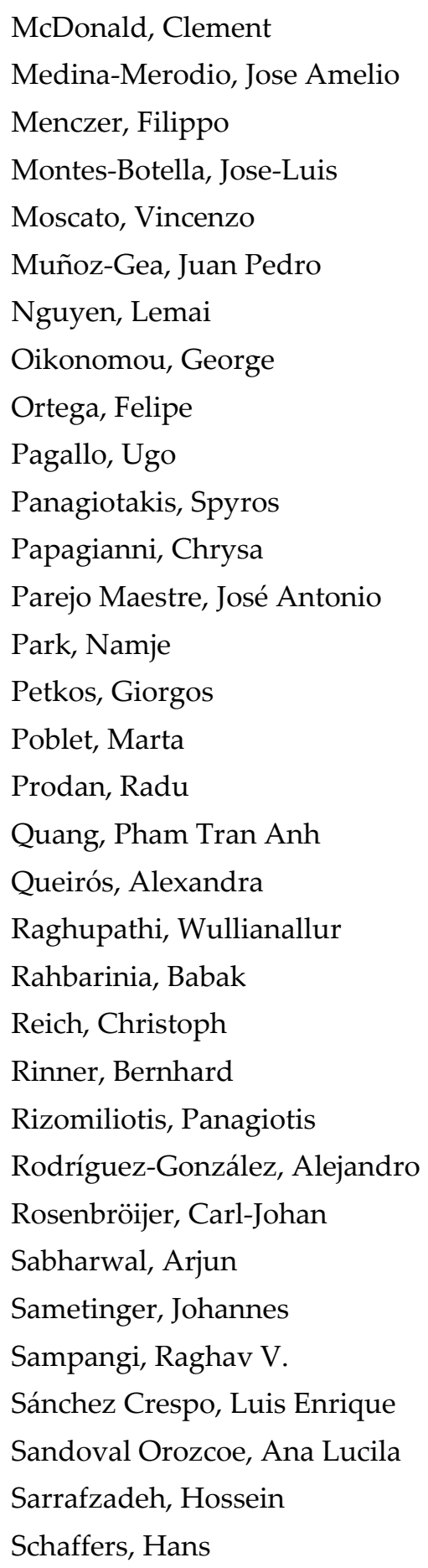

Schreiber, Fabio A.

Schuurman, Dimitri

Serôdio, Carlos

Shah, Chirag

SHEN, Lijun

Skopp, Nancy A.

So, Jungmin

Stamatiou, Yannis C.

Stantchev, Vladimir

Stiakakis, Emmanouil

Tammaro, Anna Maria

Taylor, Nick

Tervonen, Jouni K.

Torres-Sospedra, Joaquin

Tryfonas, Theo

Tsakalidis, Athanasios

Tye, Michelle

Udo, Henk

Usman, Muhammad

Valera, Alvin C.

Vamvoudakis, Kyriakos G.

Varela, Ángel

Varlamis, Iraklis

Varvarigou, Theodora A.

Vasilakos, Athanasios

Vernadat, Francois B.

Vlamos, Panayiotis

Vozniuk, Andrii

Wahab, Omar Abdel

Wang, Wei-Chih

Weber, Ingmar

Weippl, Edgar

Xiao, Liangliang

(C) 2017 by the authors; licensee MDPI, Basel, Switzerland. This article is an open access article distributed under the terms and conditions of the Creative Commons Attribution (CC-BY) license (http://creativecommons.org/licenses/by/4.0/). 